revue Recherche en Sciences de Gestion-Management Sciences-Ciencias de Gestión, n ${ }^{\circ} 137$, p.

\title{
Les paradoxes dans un établissement de santé : reconstruire du sens par l'innovation managériale
}

\author{
Anne Albert-Cromarias \\ Enseignant-Chercheur \\ Groupe ESC Clermont - CleRMa \\ (France) \\ Catherine Dos Santos \\ Enseignant-chercheur \\ Groupe ESC Clermont - CleRMa \\ (France)
}

Les établissements de santé connaissent depuis plusieurs années un tournant gestionnaire qui se traduit par une recherche de rationalisation et un risque de focalisation sur la dimension économique. Dans ce contexte, ils font face à des tensions paradoxales qui mettent à mal le sens de leur action. Notre problématique est alors la suivante: peut-on répondre aux paradoxes d'un établissement de santé par l'innovation managériale en s'appuyant sur la (re)construction de sens? La recherche s'appuie sur l'étude d'un Centre de Lutte Contre le Cancer (CLCC) et est fondée sur une analyse documentaire et des entretiens à visée compréhensive. Les résultats montrent que l'innovation managériale comme réponse aux paradoxes permettrait de recréer collectivement du sens.

Mots-clés : CLCC-Paradoxes - Innovation managériale - Création de sens.

For several years, healthcare institutions have been experiencing a managerial trend that has led to an increasing rationalisation and a risk of focusing on just the economic dimension. In such a context, they face paradoxical tensions that affect the sense of 
their action. Our question is then as follows: can the paradoxes of a healthcare establishment be addressed through managerial innovation by rebuilding sense? The research is based on the case study of a Cancer Control Unit (CCU) and is carried out through a desk review and comprehensive interviews. The results show that managerial innovation as a response to paradoxes would make it possible to collectively recreate sense.

Key-words: $C C U$ - Paradoxes - Managerial innovation Sensemaking.

Desde hace varios años, los establecimientos sanitarios conocen a un punto de inflexión en su gestión que se traduce por una búsqueda de racionalización y un riesgo de focalizarse sobre la dimensión económica. En este contexto, se confrontan a tensiones paradójicas que pueden dañar el sentido de su acción. Nuestra problemática es la siguiente: ¿podemos responder a las paradojas de un establecimiento sanitario a través de la innovación en la gestión apoyándose en la re(construcción) del sentido. La investigación se basa en el estudio de un Centros de Lucha contra el Cáncer (CLCC) y se funda en un análisis documentario y de entrevistas exhaustivas. Los resultados muestran que la innovación en gestión como respuesta a las paradojas permitiría volver a dar sentido colectivamente

Palabras-clave: CLCC - Paradojas - Innovación en gestión - dar sentido.

\section{Introduction}

Les organisations doivent répondre à des demandes simultanées et contradictoires, à l'origine de tensions pouvant être assimilées à des paradoxes (Lewis, 2000 ; Smith et Lewis, 2011 ; Jarzabkowski et al., 2013); parvenir à y répondre s'avère une condition de survie (Smith et Lewis, 2011).

Les établissements de santé, notamment, font face à des tensions entre règles et procédures formelles d'une part, et flexibilité des réponses que sous-entend l'exercice professionnel d'autre part. Par conséquent, c'est dans l'arbitrage entre routine sécurisante et flexibilité adaptative que réside l'essentiel du travail d'organisation au quotidien 
dans les services de soins. Les tensions revêtent ainsi un caractère d'injonction perçue comme paradoxale et sont facteurs de dysfonctionnements. Le recours à une démarche gestionnaire n'est pas toujours vécu comme porteur de sens entraînant une banalisation de la gestion des soins et du fonctionnement de l'organisation.

Notre recherche s'intéresse donc à la problématique des paradoxes à travers la construction de sens. En effet, dans un contexte paradoxal, la création de sens est une activité vitale (Vandangeon et Autissier, 2006). Le paradoxe crée de l'ambiguïté, de la complexité et de l'équivocité, à laquelle les managers font face en s'engageant dans un processus de construction de sens (Balogun et Johnson, 2004) qui, même s'il ne permet pas de saisir toute la complexité de la situation, leur permet de développer une compréhension négociée et ainsi d'envisager l'action (Lüscher et Lewis, 2008). Dans ce contexte, nous nous interrogeons sur le rôle que pourrait tenir l'innovation managériale (IM), qui consiste à modifier substantiellement les structures traditionnelles de l'entreprise ou la façon dont sont effectuées les tâches de management, lui permettant de mieux atteindre ses objectifs. Ainsi notre question de recherche est la suivante: face aux paradoxes à l'œuvre dans les établissements de santé et la perte de sens qui en découle, dans quelle mesure l'IM peut-elle contribuer à reconstruire du sens?

Attendu que les réformes (et les paradoxes qui en découlent) s'imposent à tous les établissements de santé, nous avons orienté notre recherche sur les Centres de Lutte Contre le Cancer (CLCC). En effet, les CLCC ont su développer un modèle innovant et efficace dans la prise en charge du cancer, modèle aujourd'hui malmené par des injonctions paradoxales. Cet article s'appuie sur les travaux d'AlbertCromarias et al. (2018) portant sur l'analyse des paradoxes au sein d'un CLCC français. Notre étude de cas, de type rechercheaccompagnement, a été conduite à partir d'une méthodologie compréhensive fondée sur une analyse documentaire et des entretiens semi-directifs.

L'article se subdivise en quatre parties. La première aborde le cadrage théorique et la problématique de la recherche. La seconde aborde l'ingénierie de la recherche : le cas et la méthodologie. Les résultats sont présentés dans la troisième. La quatrième, enfin, propose 
une analyse et une discussion des résultats dans le but d'en tirer des savoirs actionnables à la fois sur le plan théorique et pratique.

\section{1. - L'innovation managériale : pour donner du sens aux paradoxes ?}

La revue de la littérature s'intéresse, tout d'abord, aux tensions paradoxales (1.1) appelant à la (re)construction de sens (1.2) afin de s'interroger sur l'IM comme réponse pour (re)construire du sens (1.3).

\subsection{Des tensions paradoxales...}

Dans le domaine de la santé, l'affrontement entre logique quantitative (approche marchande et managériale) et qualitative (bienêtre public) aboutit à un monde paradoxant (Fortier, 2013) amenant à une polysémie des discours. En effet, ce double mouvement donne lieu à des paradoxes qui mettent à mal l'articulation entre ces régulations venues d'en « haut » (niveaux national et régional) et les dynamiques de terrain : performance économique versus qualité; cadres réglementaires et normatifs versus personnalisation de la prise en charge ; cloisonnement professionnel et institutionnel versus continuité de l'intervention auprès des patients et usagers ...

Pour autant, les notions de dilemmes, tensions, ou paradoxes ont longtemps fait l'objet d'un flou définitoire (Guédri et al., 2014). Smith et Lewis (2011, p. 382) définissent les tensions comme «des éléments qui semblent logiques pris séparément mais qui présentent des incohérences lorsqu'ils sont associés » et proposent de distinguer parmi elles trois oppositions que sont les dilemmes, les dialectiques et les paradoxes. Si dans un dilemme, un choix doit être fait entre des options tout autant désirables ou indésirables, dans une dialectique, des éléments contradictoires (thèse et antithèse) sont réunis à l'issue d'un processus d'intégration (synthèse). Le paradoxe, quant à lui et à la différence des deux précédentes oppositions, implique la présence simultanée d'éléments contradictoires pour lesquels aucun choix ne doit être effectué. A partir de cette distinction, adopter une approche paradoxale permet d'accepter les tensions en faisant coexister leurs éléments constitutifs (paradoxe) plutôt que de les résoudre en faisant disparaître l'un des composants de la tension (dilemme) ou en les noyant dans un concept englobant qui masque les oppositions (dialectique) (Luscher et Lewis, 2008). 
Alors que la littérature s'est efforcée de bâtir une typologie des différents paradoxes identifiables dans une organisation (Schad et $a l$., 2016), nous retiendrons ici les quatre catégories de Smith et Lewis (2011), à savoir d'apprentissage (learning paradoxes), d'appartenance (belonging), de gestion (organizing) et d'exécution (performing) : (1) le paradoxe d'apprentissage lorsque le système organisationnel est amené à évoluer et à se renouveler. Les tensions inhérentes à ces paradoxes découlent de la difficulté de s'appuyer sur le passé tout en le modifiant pour créer le futur ; (2) le paradoxe d'appartenance, situé entre l'individu et le collectif, qui survient lorsque les acteurs et groupes d'acteurs poursuivent à la fois l'homogénéité et la distinction; (3) le paradoxe de gestion, caractéristique des situations où un même objectif peut être obtenu en empruntant des voies différentes ; (4) le paradoxe d'exécution, issu de la pluralité des parties prenantes qui poursuivent chacune des objectifs spécifiques.

Si les travaux de recherche portant sur les modes de gestion des paradoxes ou leurs modes d'usage dans les dynamiques managériales et organisationnelles sont plus rares, la littérature s'accorde aujourd'hui à considérer qu'il est contre-productif de vouloir faire disparaître les paradoxes car ceux-ci permettent d'initier une dynamique de changement (Lewis, 2000 ; Josserand et Perret, 2003), sous réserve de trouver la bonne méthode pour tendre vers le cercle vertueux et éviter le cercle vicieux (Lewis, 2000). Lewis (2000) identifie trois mécanismes interreliés de gestion des paradoxes : l'acceptation, la confrontation ou la transcendance, cette dernière

renvoyant à la capacité à penser de manière paradoxale, c'est-à-dire à aller au-delà des contradictions et à se fonder sur elles pour évoluer. Les paradoxes apparaissent donc à la fois comme des opportunités et des menaces, selon les circonstances. On retrouve l'idée d'une organisation chaotique régie par des forces contraires qui peuvent mener soit à l'ordre, soit au chaos. Dans ce contexte, la recherche du bon équilibre entre ses forces opposées devient la clé du succès organisationnel. Smith et Lewis (2011) évoquent le fait que les organisations doivent s'efforcer de faire émerger le «potentiel positif » des paradoxes.

\subsection{Un contexte appelant à la création de sens}

La profusion des situations paradoxales crée un état de confusion cognitive qui entrave l'élaboration de repères pour l'action et compromet (provisoirement) la capacité des acteurs à coordonner leurs 
systèmes d'action (Weick, 1995). Mais dans le même temps, par les multiples interrogations qu'elle suscite à tous les niveaux de la hiérarchie, l'équivocité du contexte déclenche une volonté de créer du sens ; elle stimule les échanges de points de vue entre les acteurs en quête de sens, et constitue alors un contexte favorable à l'éclosion du sensemaking, qui consiste à créer une vision co-construite par toutes les parties en présence à partir de leurs propres représentations. Cette approche développée par Weick (1995) cherche à savoir comment les individus s'entendent sur ce qu'ils doivent faire, de manière à pouvoir se coordonner pour la réalisation de l'action (Vandangeon et Autissier, 2006).

La théorie du sensemaking se développe plus particulièrement dans les situations complexes ou incertaines, voire paradoxales, nécessitant une participation de tous les acteurs pour obtenir un point de vue fidèle à la réalité des situations possibles.

Ainsi, l'activité de création de sens repose sur deux dimensions :

- La dimension sociale, la création de sens étant un processus individuel ou collectif.

- La dimension organisationnelle, reposant sur une démarche intra ou inter-organisationnelle.

Lesca et Chokron (2002) démontrent qu'une démarche autonome de création de sens, forcément limitée et très fragmentaire, est moins pertinente qu'une approche collective. En effet, le raisonnement personnel est, d'une part, contraint par sa propre rationalité limitée (Simon, 1955) et, d'autre part influencé par des biais cognitifs contingents à l'individu (Jaouen et Tessier, 2008).

La création de sens peut être tournée vers l'intérieur de l'entreprise (démarche intra-organisationnelle). Lesca et Lesca (2009) identifient une co-construction de sens lorsque (1) les participants font émerger du sens auquel ils n'avaient pas abouti individuellement, ou (2) l'un des participants ayant perçu le sens possible des signaux faibles rallie l'ensemble des membres à sa propre perception.

Dans certaines situations, cette forme d'intelligence collective semble ne pas suffire pour appréhender la complexité de l'environnement et de ses évolutions, c'est pourquoi une démarche inter-organisationnelle constitue l'ultime évolution. Les acteurs 
nourrissent alors leur perception stratégique d'interactions sociales au gré de leurs rencontres et discussions avec les parties prenantes.

En croisant les dimensions sociale et organisationnelle, Lafaye et Berger-Douce (2012) aboutissent à une typologie de la création de sens stratégique (tableau 1).

\section{Tableau 1. Typologie de la création de sens stratégique}

\begin{tabular}{|l|l|l|}
\hline $\begin{array}{c}\text { Dimension } \\
\text { organisationnelle }\end{array}$ & $\begin{array}{l}\text { Intra- } \\
\text { organisationnelle }\end{array}$ & $\begin{array}{l}\text { Inter- } \\
\text { organisationnelle }\end{array}$ \\
$\begin{array}{l}\text { Dimension } \\
\text { sociale }\end{array}$ & (1) Isolée & (2) Alimentée \\
\hline Individuelle & (3) Partagée & (4) Mutualisée \\
\hline Collective & (4) \\
\hline
\end{tabular}

Source : Lafaye et Berger-Douce, 2012, p. 15.

Puisqu'il est admis que le résultat qualitatif de l'intelligence collective est supérieur à celui de l'intelligence individuelle, nous pouvons analyser les diverses situations de la manière suivante :

- Quadrant 1: l'interprétation d'une seule personne sans enrichissement social rend la création de sens moins pertinente.

- Quadrant 2 : l'interprétation est toujours le fruit d'une seule personne mais elle a été nourrie d'échanges avec d'autres organisations.

- Quadrant 3: le processus de création de sens devient collectif puisqu'il s'appuie sur des échanges de plusieurs individus d'une même entreprise.

- Quadrant 4 : les échanges sont plus étendus encore puisqu'ils concernent plusieurs individus appartenant à des organisations 
distinctes. Cette situation est la plus favorable mais également la plus complexe à mettre en œuvre.

\subsection{L'innovation managériale : reconstruire du sens face aux paradoxes?}

Dans un monde paradoxant appelant à une (re) construction de sens, l'élaboration de stratégies innovantes est admise comme un exercice particulièrement complexe, la concurrence rendant instable, voire même hostile, l'environnement. Cependant, dans une logique évolutionniste, l'établissement de santé ne peut échapper à la régulation de son champ organisationnel, pouvant parfois imiter (stratégies d'évitement qui sont rarement productives sur le long terme), mais le plus souvent contraint d'innover au risque de disparaitre, et se doit donc de transformer ses pratiques managériales. En effet, la diversité des enjeux et attentes ne manque pas de multiplier, dans l'activité, des tensions contradictoires qui obligent les acteurs à effectuer un travail important de gestion des décalages entre le prescrit et le réel. Cette évolution rend plus que jamais sensible la question du management, et des pratiques innovantes, visant à arbitrer entre les objectifs et à construire des compromis.

La littérature sur l'IM fournit quelques repères pour mieux en comprendre l'implication en milieu hospitalier.

Birkinshaw et al. (2008, p.829) définissent l'IM par « ce qui modifie substantiellement la façon dont les tâches de management sont effectuées et/ou les structures traditionnelles de l'organisation lui permettant ainsi de mieux atteindre ses objectifs ». L'IM prend ainsi la forme de nouveaux dispositifs de management, de nouveaux outils de gestion ainsi que de nouvelles pratiques et se traduit par de nouvelles formes organisationnelles transformant les structures.

Le Roy et al. (2013) considèrent deux modalités pour définir l'IM. La première fait référence à «l'intention et l'adoption d'une pratique ou d'une méthode de management complètement nouvelle par rapport aux pratiques existantes »; la seconde est définie par «l'adoption pour une organisation de pratiques nouvelles par rapport à ses pratiques existantes $»$. Cette double modalité amène à différencier, dans un premier temps, des innovations reposant sur un processus de création ex-nihilo et, dans un second temps, des 
innovations consistant à transférer dans un contexte nouveau une innovation déjà expérimentée ailleurs.

Les recherches sur l'IM en milieu hospitalier n'ont certes pas fait l'objet de travaux conséquents, mais Nobre (2013), à partir d'IM expérimentées, identifie trois logiques à l'origine de ces dernières :

- L'IM par la création de nouvelles entités pour combler un manque de coordination organisationnelle: il s'agit de renforcer, voire de créer, le lien pour développer un processus nécessitant la coordination de différents acteurs qui étaient au préalable sans connexion.

- L'IM par le développement de nouvelles relations organisationnelles : l'idée est de faire évoluer les relations entre les acteurs, au sein de l'établissement mais également avec l'externe.

- L'IM par la transformation organisationnelle pour adapter les structures: le but est de transformer l'infrastructure ou l'articulation des directions afin qu'elles répondent mieux aux besoins de prise en charge du patient par exemple.

Les IM concernent donc davantage la dimension structurelle : il s'agit de créer du lien organisationnel et de la coordination entre les mondes sociaux afin de clarifier la prise de décision et responsabiliser les acteurs, ce que Claveranne et Christophe (2004) qualifient de «faire-ensemble». Nobre (2013) considère ainsi que les IM sont finalement les réponses apportées par les établissements pour dépasser les difficultés liées aux caractéristiques ontologiques de l'hôpital : le cloisonnement, la diversité des acteurs et la complexité des rapports de pouvoir.

Notre recherche s'attache à répondre à la question suivante : face aux paradoxes à l'œuvre dans les établissements de santé et la perte de sens qui en découle, l'IM peut-elle contribuer à reconstruire du sens?

\section{2. - Les paradoxes d'un Centre de Lutte Contre le Cancer}

\subsection{Le contexte : les réformes de la santé en France}


Depuis une vingtaine d'années, les réformes du système hospitalier français oscillent entre deux référentiels : l'intervention croissante des pouvoirs publics dans la gestion des établissements de soins ou le marché comme seul moyen de coordination des acteurs.

Avec les ordonnances dites Juppé de 1996, le secteur hospitalier se caractérise par une intervention croissante de l'État. La création des agences régionales de l'hospitalisation (ARH) symbolise cette nouvelle logique : en tant que groupements d'intérêt public, elles centralisent des pouvoirs qui dépendaient auparavant des Directions régionales de l'action sanitaire et sociale (DRASS) et leurs déclinaisons départementales (DDASS). Leur objectif est de rationaliser la politique hospitalière régionale, notamment par la mise en commun des activités publiques et privées (Tabuteau, 2013). Désormais, dans la perspective du New Public Management, la contractualisation entre la tutelle et les établissements hospitaliers devient le one best way de la politique hospitalière (Ruellan, 2015).

Les évolutions réglementaires du système hospitalier français vont également, depuis plusieurs années, dans le sens d'un rapprochement de leurs modèles organisationnels et managériaux avec ceux du privé dans le but d'améliorer leur performance économique. Deux réformes majeures symbolisent cette tendance : d'une part, la loi de financement de la sécurité sociale de 2003, dite «T2A » («tarification à l'activité»), qui a entraîné un changement de financement des établissements hospitaliers visant à instaurer un tarif identique dans tous les établissements publics ainsi qu'une convergence avec le secteur privé permettant ainsi de mieux comparer les coûts d'hospitalisation entre les deux secteurs et d'attiser la concurrence entre établissements de santé (Zeynep, 2014 ; Burnel, 2017). D'autre part, la loi de 2009 portant réforme de l'hôpital et relative aux patients, à la santé et aux territoires, dite «HPST », qui a entraîné une restructuration profonde des hôpitaux afin d'accompagner les changements allant dans le sens de pratiques gestionnaires par une organisation idoine avec une gouvernance hospitalière nouvelle.

\subsection{Présentation du cas}

Notre étude porte sur un CLCC, Etablissement de Soins Privés d'Intérêt Collectif (ESPIC) à but non lucratif, qui assure, sous statut privé, une mission de service public hospitalier. Il a été fondé en 1973, 
compte actuellement 750 collaborateurs (dont 180 médecins et internes) pour une activité annuelle de plus de 4500 interventions sur six salles de bloc opératoire. Intervenant plus spécifiquement sur les cancers du poumon, du sein et de la thyroïde, il bénéficie d'une expertise reconnue en radiothérapie et médecine nucléaire.

Installé sur le site du CHU, avec lequel il partage une mission de service public, il entretient depuis sa création une relation complexe avec ce partenaire, marquée par une peur récurrente de se faire absorber. Localement, on compte par ailleurs deux grosses cliniques privées, Clinique 1 créée en 1974 et Clinique 2 depuis 1987. Sur la région, existent également plusieurs petits établissements publics ou privés.

Tableau 2. Quelques chiffres de l'activité des principaux acteurs locaux (2016)

\begin{tabular}{|c|c|c|c|c|}
\hline \multirow{2}{*}{} & \multicolumn{3}{|l}{ Nombre d'actes ou séances $\mathrm{MCO}^{1}$} \\
\cline { 2 - 5 } & CLCC & CHU & \multicolumn{1}{l|}{$\begin{array}{l}\text { Clinique } \\
\mathbf{1}\end{array}$} & $\begin{array}{l}\text { Clinique } \\
\mathbf{2}\end{array}$ \\
\hline $\begin{array}{l}\text { Nombre total } \\
\text { d'actes ou } \\
\text { séances }\end{array}$ & 54268 & 120352 & 32566 & 35510 \\
\hline \multicolumn{4}{|c|}{ En cancérologie (quelques exemples) } \\
\hline Pneumologie & 978 & 446 & 4 & 58 \\
\hline $\begin{array}{c}\text { Appareil } \\
\text { digestif }\end{array}$ & 114 & 625 & 68 & 109 \\
\hline Sénologie & 1241 & 358 & 347 & 1043 \\
\hline Thyroïde & 347 & 228 & 3 & 58 \\
\hline Urologie & 36 & 337 & 101 & 46 \\
\hline Gynécologie & 225 & 1280 & 981 & 174 \\
\hline Chimiothérapie & 11985 & 19494 & 985 & 11399 \\
\hline Radiothérapie & 13335 & - & - & - \\
\hline
\end{tabular}

\footnotetext{
${ }^{1}$ MCO : Médecine Chirurgie Obstétrique, y compris activité ambulatoire et cancérologie.
} 
Source : ATIH, données ScanSanté Casemix MCO

Depuis cinq ans, le CLCC est confronté à un changement brutal d'environnement. Il entretenait une relation relativement hostile avec le CHU, collaborait convenablement avec la Clinique 1, qui ne possède pas de service de radiothérapie, et s'affrontait davantage avec l'autre grand acteur privé (Clinique 2) qui possède des oncologues et pratique également la chirurgie du sein. Il y a quatre ans, le projet de GHT a renforcé l'autorité du CHU sur le département et les trois départements voisins. Par ailleurs, depuis 2017, le contexte local a été profondément modifié puisque les deux principales cliniques privées du territoire font désormais partie du même groupe.

\subsection{Cadrage méthodologique de la recherche}

Notre recherche, débutée au printemps 2016, s'inscrit dans une démarche compréhensive au sens de Dumez (2013). Elle cherche à décrire et expliquer un phénomène à partir d'un cadre conceptuel, reposant ici sur l'IM au sens de Nobre (2013), permettant, d'une part, de répondre aux paradoxes et, d'autre part, de créer collectivement du sens. Cette recherche s'appuie sur une étude de cas unique qui nous permet d'aller vers la compréhension d'un phénomène complexe dans son contexte d'ancrage. Cette unicité n'entrave pas selon nous la portée de cette étude de cas, dans la mesure où l'objectif est de mettre à jour des phénomènes dont la compréhension peut enrichir la théorie (Gombault, 2005). S'agissant d'une recherche à dominante exploratoire en raison du caractère émergent du thème, une méthodologie qualitative (Wacheux, 1996) semblait la plus appropriée pour mieux identifier les paradoxes et les innovations managériales déployées en réponse et ainsi saisir les mécanismes de création de sens stratégique.

La démarche est articulée essentiellement autour de deux sources d'informations : l'analyse documentaire et la réalisation d'entretiens semi-directifs menés en face-à-face. La collecte et la prise de connaissance des documents internes et externes relatifs au contexte ont permis une meilleure compréhension de la problématique et facilité la réalisation de la mission. La démarche a consisté à repérer des mots clés (à partir de 23 documents analysés par le logiciel Tropes) afin d'illustrer des paradoxes et les IM à l'œuvre. Des entretiens semidirectifs ont été menés auprès de treize acteurs clés au sein de l'organisation (personnel médical, personnel soignant, fonctions 
supports) et de neuf parties prenantes externes, partenaires du CLCC, à la fois dans des relations de concurrence et de coopération (cf. tableau $3)$.

Tableau 3. Entretiens menés

\begin{tabular}{|c|c|c|}
\hline Etablissement & $\begin{array}{c}\text { Catégorie de } \\
\text { personnel }\end{array}$ & Nombre \\
\hline CLCC & $\begin{array}{c}\text { Médical : } 4 \\
\text { Soignant : 4 } \\
\text { Support : 5 }\end{array}$ & 13 \\
\hline $\begin{array}{c}\text { Hospitalisation à } \\
\text { Domicile (HAD) }\end{array}$ & $\begin{array}{c}\text { Médical : 1 } \\
\text { Soignant : 1 } \\
\text { Support : 1 }\end{array}$ & 3 \\
\hline $\begin{array}{c}\text { Réseau régional de } \\
\text { cancérologie }\end{array}$ & Médical : 1 & 1 \\
\hline $\begin{array}{c}\text { Clinique privée 1 } \\
\text { Médical : 1 } \\
\text { Support :1 }\end{array}$ & 2 \\
\hline $\begin{array}{c}\text { Clinique privée 2 } \\
\text { Autres experts (hors } \\
\text { régions) }\end{array}$ & Support : 1 & 1 \\
\hline \multicolumn{2}{|c|}{ Total } & $\mathbf{2 2}$ \\
\hline
\end{tabular}

Source : auteurs

Ces entretiens ont été menés sur la base d'un guide comprenant quatre grandes thématiques ( $1 /$ contexte du CLCC $; 2 /$ paradoxes auxquels il est confronté ; 3/ quelle(s) IM face à ces paradoxes ; 4/ pour quelle construction de sens ?) à raison d'une heure environ par interviewé. Ces entretiens ont été enregistrés puis retranscrits intégralement pour exploitation verticale et horizontale, dans une logique de codage à partir des mots clés identifiés dans la phase d'analyse documentaire. Enfin, une triangulation des données primaires et secondaires a permis de formuler les paradoxes et de repérer les réponses apportées. 


\section{3. - Résultats : l'innovation managériale comme réponse à la perte de sens induites par les paradoxes.}

Les quatre catégories de paradoxes identifiées par Smith et Lewis (2011) sont repérables au sein d'un CLCC (Albert-Cromarias et $a l ., 2018$ ). En effet, le paradoxe d'apprentissage se manifeste au travers de la dualité qu'imposent la concurrence et la coopération entre établissements. Le paradoxe d'appartenance s'exprime dans l'opposition entre valeurs caritatives et efficience gestionnaire ainsi que dans la cohabitation, parfois difficile, des différents mondes sociaux : médical, soignant et administratif. Le paradoxe de gestion s'impose lorsqu'il s'agit d'arbitrer entre santé et logique gestionnaire, lorsqu'il s'agit de concilier les injonctions externes quant à la qualité des soins et leur mise en œuvre, ou encore lorsqu'il faut considérer les spécificités liées à l'activité de cancérologie. Enfin, le paradoxe d'exécution prend place lorsque le personnel est centré sur des problèmes opérationnels internes au détriment des patients ou lorsque s'expriment des jeux d'acteurs.

Les paradoxes identifiés déconstruisent du sens dans la mesure où ils ne mettent pas systématiquement en cohérence les valeurs et les pratiques de gestion et créent un cloisonnement entre les différents acteurs, susceptibles de fragiliser leurs liens. En effet, les répercussions du tournant gestionnaire sur le travail se révèlent à travers la perte de sens dont il a pu être porteur au profit d'une focalisation sur les objectifs financiers et d'une distanciation entre gestionnaires et personnels soignants, les premiers ayant accès aux réalités de l'activité des seconds au prisme des indicateurs fournis par les outils de gestion.

Nos observations au sein du CLCC étudié montrent qu'en réponse aux injonctions paradoxales, des démarches d'IM s'inscrivant dans les trois logiques de Nobre (2013), sont entreprises. Différents acteurs en sont à l'origine (directeur d'établissement, directeurs fonctionnels, membres du corps médical) et fondent leur action sur la conviction profonde de la nécessité d'introduire de nouvelles approches et de nouvelles méthodes afin de transformer le fonctionnement de l'établissement, dans une logique de (re)création de sens.

\subsection{L'IM par création de nouvelle entité : une réponse au paradoxe d'apprentissage}


Le CLCC développe aujourd'hui des projets qui se veulent mobilisateurs. Ainsi la création d'un Institut du Sein (IS), avec pour objectif d'être "présent sur toute la filière, depuis le dépistage systématique jusqu'au palliatif » [5] est en projet, en partenariat avec un établissement privé local. Particulièrement délicat à mettre en œuvre, puisqu'il s'agit de monter une unité de soins commune, en partageant les activités, le projet semble malgré tout mobilisateur, notamment dans sa dimension novatrice (création d'un univers virtuel pour le patient, création d'une marque commune). Etonnamment, il semble fédérer plus facilement qu'un projet a priori plus simple comme la mutualisation d'un équipement lourd de type scanner ou IRM.

Ici, le paradoxe d'apprentissage «concurrence / coopération » va être utilisé par les dirigeants dans un discours faisant de la concurrence un outil d'innovation : "on motive par du nouveau, de l'inédit, une utopie mobilisatrice » [11]. Les dirigeants affirment également la nécessité d'une démarche proactive de coopération avec le privé : "la prise en charge du cancer du sein est un processus complexe, associant des activités aussi différentes que le dépistage systématique, le diagnostic de cas plus ou moins graves, la chirurgie, la radiothérapie, la chimiothérapie, l'accompagnement aux soins de supports, ... Aussi, nous ne pouvons pas tout faire, ni ignorer la progression du privé sur les créneaux les plus rentables, donc autant coopérer et prendre en main la coordination de cette activité sur le territoire " (discours des dirigeants, 2015). Le projet est ainsi vu comme clé potentielle de succès de développer une plateforme d'accueil et de gestion des cas qui donneront au CLCC autorité sur la régulation et la gestion de la filière de soins sur tout le territoire.

Ainsi, l'IM, en la création d'une nouvelle entité qu'est l'IS, permet de trouver une réponse au paradoxe d'apprentissage, découlant de l'affrontement des logiques concurrentielles et coopératives. Cette IM permet au CLCC de mieux remplir sa mission stratégique tout en mutualisant ses ressources. Cette concentration des activités relevant du cancer du sein (du dépistage au traitement) permet ainsi une convergence entre projet stratégique et projet thérapeutique.

\subsection{L'IM par développement de nouvelles relations : une réponse aux paradoxes d'appartenance et de gestion}


Dans notre cas, le paradoxe d'appartenance relevé montre l'opposition entre discours expert et discours stratégique. Les valeurs des CLCC (non-lucrativité, absence de dépassements d'honoraires, action médicale désintéressée et motivée par la mission de service public) s'opposent à la logique d'efficience gestionnaire des dirigeants, construisant en interne même des contradictions. La prise en compte du paradoxe semble ici relativement peu développée, les acteurs internes étant simplement conscients de la situation. En l'absence de représentation permettant à chacun des mondes de penser la cohabitation entre contrainte d'efficience et mission de service non lucrative, ne faudrait-il pas chercher la structuration d'une telle organisation pluraliste autour d'une stratégie capable de prendre en compte les attentes spécifiques aux mondes gestionnaire et médical en présence?

Face au paradoxe d'appartenance, le CLCC met en place une démarche d'IM reposant sur l'évolution des relations entre les acteurs internes. Cette démarche concerne plus particulièrement les cadres de santé qui occupent une place centrale dans la problématique organisationnelle de l'établissement. Néanmoins, leur déficit de légitimité, notamment par rapport au corps médical, et leur fort ancrage dans le soin constituent un obstacle pour leur appropriation des problématiques stratégiques et managériales. Le dispositif mis en œuvre par le CLCC repose sur une méthodologie de groupes de travail intra-organisationnels impliquant l'ensemble des cadres, avec pour objectif d'identifier les principaux défis et enjeux managériaux afin de formuler des réponses et élaborer des outils ad hoc. La démarche concerne donc aussi bien la définition du management que son déploiement en adéquation avec les orientations stratégiques de l'établissement.

Le CLCC déploie également une démarche d'IM reposant sur l'évolution des relations entre les acteurs, cette fois-ci externes, répondant ainsi au paradoxe de gestion. Conscient de l'importance de la prise en charge du cancer sur le territoire régional et de la dispersion des acteurs régionaux, le CLCC encourage les collaborations (avec des établissements publics d'autres communes ou des établissements privés) permettant ainsi de fournir une réponse adaptée et innovante aux patients et une plus forte synergie entre l'ensemble des acteurs. 


\subsection{L'IM par transformation organisationnelle : une réponse au paradoxe d'exécution}

Sous la contrainte économique mais également la capacité d'accueil des patients, le CLCC limite la durée d'hospitalisation d'un patient et privilégie souvent la chirurgie ambulatoire. En effet, il s'avère moins coûteux et moins pénalisant pour le CLCC de privilégier l'ambulatoire plutôt que l'hospitalisation, quitte à payer au patient qui vient de loin une nuit d'hôtel, ce qui constitue un paradoxe d'exécution.

Afin de satisfaire aux besoins de prise en charge et de santé du patient, la formation des infirmiers s'avère indispensable. Mais le CLCC demeure contraint par une activité importante et des effectifs limités. Répondre à ce paradoxe d'exécution passe par convaincre les gestionnaires que la formation des infirmiers peut avoir à long terme un effet sur la réduction des coûts et/ou sur l'augmentation de l'activité rémunérée.

Ici, le CLCC répond par l'IM en transformant l'organisation afin d'adapter la structure. En effet, afin de faire face aux contraintes économiques tout en satisfaisant les besoins de santé des patients, l'établissement restructure ses services en fonction du parcours patient en privilégiant le développement de nouveaux processus de prise en charge (ambulatoire, etc.). Cette transformation est permise grâce, d'une part, au comportement de la direction qui s'implique dans la prise en charge du patient (notamment via des questionnaires de satisfaction) et gagne en légitimité par une présence et une immersion sur le terrain, et d'autre part, à l'appui des cadres pour concevoir et mettre en œuvre le changement après concertation avec le corps médical.

Les paradoxes se produisent à tous les niveaux d'interactions sociales : entre les organisations mais aussi entre des individus ou groupes d'individus au sein d'une ou de plusieurs organisations. Mais au-delà de cette variété, le contexte du CLCC suggère une dimension universelle dans la problématique du management de ces paradoxes : quel que soit le statut des parties prenantes impliquées en termes de pouvoir, centralité et légitimité, ces dernières sont amenées à gérer les paradoxes et à penser un moyen de sortir de la contradiction qui passe par l'IM. 


\section{4. - Discussion: management des paradoxes dans une} logique de création de sens : l'innovation managériale au service du dialogue et du collectif

De notre étude, il ressort que la gestion des paradoxes relève

d'une habileté managériale (Grenier, 2014) pour permettre un apprentissage collectif réciproque des acteurs et la construction de sens (Grenier et Ibrahim, 2015), dans une logique d'IM comme définie par Birkinshaw et al. (2008) et Le Roy et al. (2013) (figure 1). 


\section{Figure 1. L'innovation managériale au service du dialogue et du collectif}

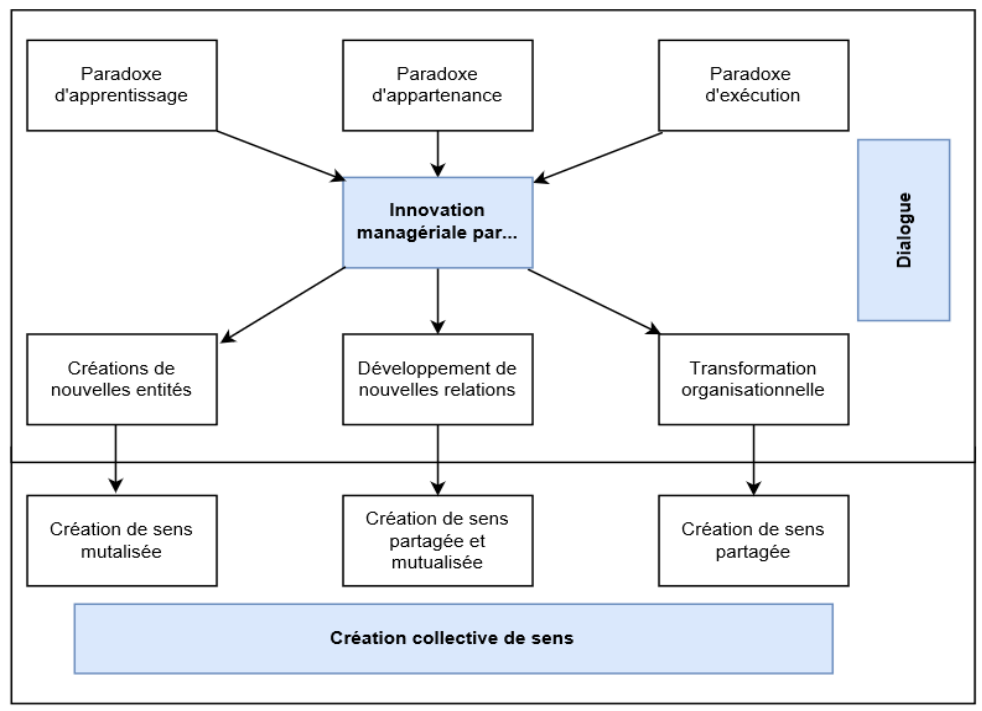

Source : auteurs

\subsection{L'IM par le dialogue}

Dans une perspective évolutionniste, l'IM joue un rôle déterminant pour engendrer une dynamique de transformation des routines de l'organisation et sortir ainsi d'une forme de déterminisme. Finalement, il n'est pas seulement question de résoudre les paradoxes, mais aussi de les entretenir, ou de les transcender, en tirant parti de leur capacité à entretenir la dynamique des organisations. Dans ce contexte, l'IM permet d'envisager d'autres modalités de coopération et de coordination entre les personnes dans un système de production contraint. Le dialogue, dans le management des paradoxes, devient ainsi central (Josserand et Perret, 2003).

Traiter les paradoxes via le dialogue permet au CLCC d'utiliser le rôle bénéfique de ces derniers en tant que régulateurs et/ou catalyseurs de la dynamique d'apprentissage de l'organisation. 
L'existence et le maintien de tensions et autres paradoxes apparaissent comme une condition favorable à la mise en œuvre d'une logique de co-construction d'une vision partagée, qui ensuite facilitera l'adoption et l'adhésion des différents acteurs. Nos résultats, en cohérence avec la littérature sur le dialogue, mettent l'accent sur le rôle de la mise en discours, de la traduction et de la narration pour tenter de légitimer des points de vue divergents et finalement de les concilier. De telles pratiques semblent particulièrement adaptées lorsque les tensions touchent la raison d'être d'une profession (les médecins), et ses interactions avec d'autres groupes (les gestionnaires). Par ailleurs, le contexte spécifique du CLCC impose un caractère partenarial de l'action, éclatée entre des acteurs multiples, internes (personnel soignant, patient, direction, etc.) et externes (Etat, région, $\mathrm{CHU}$ et cliniques privées).

Les IM intra-organisationnelles s'appuyant sur le dialogue, avec les cadres de santé notamment, sont de nature à renforcer les facteurs individuels et organisationnels qui favorisent l'acceptation des tensions (Smith et Lewis, 2011). Le dialogue induit en effet une sécurisation psychologique importante des acteurs trouvant sa source dans l'amélioration continue (résolution de dysfonctionnements tels que la conformité réglementaire, la santé-sécurité du patient, etc.) et dans la stratégie collective (échanges, confiance, entraide, sortie de l'isolement, etc.). Il rassure également la Direction qui gagne ainsi une sérénité via la confiance susceptible d'être développée. En favorisant le confort psychologique, les IM intra-organisationnelles apporteraient donc une meilleure stabilité émotionnelle, minimisant ainsi la peur et les attitudes défensives face aux tensions paradoxales.

Par ailleurs, les IM inter-organisationnelles, via le dialogue avec les établissements concurrents mais également les homologues de la région, permettent d'agir collectivement et de fait créer un mode relationnel privilégié dans le contexte économique contemporain complexe, marqué par le coût de l'innovation technologique, la recherche de réductions des dépenses de santé, ou de nouvelles formes d'organisations. 


\subsection{L'IM pour une création collective de sens}

L'IM permet également une création de sens collective, qu'elle soit « partagée » ou « mutualisée » (Lafaye et Berger-Douce, 2012).

En effet, l'IM par création de nouvelle entité (Nobre, 2013) favorise la création de sens «mutualisée » puisque les différents acteurs inscrits dans le projet commun (le projet IS dans notre cas) vont partager les informations et connaissances issues de leurs expériences mais aussi mutualiser certaines ressources humaines. Pour mobiliser le monde médical dans ce projet, et afin de s'inspirer d'une expérience réussie, un groupe de médecins et soignants a rencontré les Hôpitaux Universitaires de Genève qui ont initié la même démarche. Un tel projet s'inscrit donc dans la lignée de travaux de Weick (1995) qui prône le caractère collectif du processus et l'intérêt des débats, discussions, confrontations d'idées, ... pour créer un sens aux situations et à l'environnement.

La création de sens est également « mutualisée » grâce à l'IM par développement de nouvelles relations (Nobre, 2013) avec les acteurs du territoire. Les interactions avec d'autres établissements de prise en charge du cancer, les discussions et une interaction collective, constituent autant de moyens de faire face à l'ambigüité et l'incertitude liées aux soins de cancérologie. L'interprétation individuelle des informations collectées sur la pathologie peut parfois être subjective dans la mesure où elle est conditionnée, par exemple, par l'expérience spécifique et les connaissances propres à chacun. Le travail collectif de sens est censé réduire cette subjectivité mais aussi certains biais cognitifs individuels.

En s'appuyant sur un partage d'informations avec ses collaborateurs, la direction du CLCC s'inscrit dans une approche «partagée » de la création de sens. Les innovations managériales par développement des nouvelles relations intra et par transformation organisationnelle permettent une telle création de sens. Là encore, le collectif prime et, comme le recommandent Lesca et Lesca (2009), la direction du CLCC l'encourage : elle amène les cadres à s'interroger sur le parcours du patient, à construire un sens commun, et les encourage à s'interroger sur l'opérationnalité de leur travail. Cette posture d'accompagnement de la direction témoigne de sa capacité à ne pas orienter unilatéralement les échanges, se positionnant en tant que 
facilitateur de création de sens et s'inscrivant dans une démarche de sensemaking, plutôt que dans une démarche sensegiving.

Conformément aux travaux de Weick (1995), les différentes innovations managériales adoptées par le CLCC s'inscrivent davantage dans la création collective de sens que dans la création d'un sens collectif. Les logiques de coopération au sein des équipes projet (qu'elles soient intra ou inter-organisationnelles), en considérant les paradoxes auquel est confronté l'établissement, constituent une IM via un travail de redéfinition collectif du processus organisant. La confrontation des subjectivités, l'argumentation, le débat donnent la possibilité aux collaborateurs mais également aux acteurs externes, concurrents ou partenaires du CLCC, de parvenir collectivement à un sens de la situation. Les mécanismes d'interactions observés aboutissent effectivement au partage des interprétations, au développement d'attentes compatibles portant sur des intérêts communs et, in fine, à la stabilisation du sens. Les différents acteurs coordonnent progressivement leurs actions, laissant ainsi apparaître un nouveau système d'actions organisées.

Dans cette démarche, la direction a un rôle fondamental en créant un contexte favorable lié à une implication des acteurs internes et externes. Un réseau d'acteurs soutenant la démarche favorise l'émergence de sens commun. Cette approche managériale ascendante contribue à donner une véritable place aux acteurs du terrain. Il ne s'agit donc pas simplement d'appliquer une méthode, il s'agit également d'adopter un nouveau mode managérial, donc une innovation managériale.

\section{Conclusion}

Cette recherche visait à comprendre en quoi les réponses aux paradoxes constituent une logique d'IM (Birkinshaw et al., 2008 ; Nobre, 2013) permettant de (re)construire du sens (Grenier et Ibrahim, 2015).

Les processus d'IM étudiés au sein du CLCC s'avèrent porteurs de sens puisqu'ils permettent de mettre en cohérence les valeurs et les pratiques de gestion du CLCC. Au lieu de créer un cloisonnement entre les différentes catégories d'acteurs, ils contribuent à renforcer leurs liens. Ils s'accompagnent de la construction d'un discours sur les pratiques, porté par une logique de dialogue, tant intra 
qu'inter-organisationnel, rendant compte de l'engagement et du travail collectif réalisé. La création de sens correspond ainsi à un processus collectif (qu'il soit partagé ou mutualisé) mettant en interaction les acteurs internes et externes afin de traiter les informations en provenance de l'environnement et amener à une compréhension mutuelle des enjeux de la cancérologie dans un but d'anticipation de manière à donner de la légitimité au CLCC.

Notre recherche, fondée sur une étude de cas unique, n'est bien entendu pas exempte de biais, notamment ceux liés à la formulation des paradoxes par les interviewés eux-mêmes, ainsi qu'à l'interprétation qui en est faite par le chercheur. Il faut également considérer que plusieurs des projets évoqués n'étaient qu'au stade d'ébauche au moment de l'étude et que, par conséquent, une analyse complémentaire ultérieure devra confirmer ces premiers résultats. De plus, portant sur un CLCC particulier, il convient d'être prudent dans la généralisation des résultats aux autres CLCC, voire aux établissements de santé plus largement.

Cette recherche ouvre néanmoins des perspectives. Sur le plan théorique, elle permet d'élargir le paradoxe au-delà d'une vision traditionnelle limitée aux acteurs « entreprises » en l'élargissant au champ de la santé. Sur le plan managérial, elle permet de considérer les IM comme des réponses apportées par les établissements pour dépasser les difficultés liées aux paradoxes en vue de redonner du sens aux actions. L'objectif induit de « faire-ensemble » pose donc, en filigrane, la question du changement des pratiques de management. 
Albert-Cromarias A., Dos Santos C. \& Nautre B., «Quelle réponse stratégique aux paradoxes d'un centre de lutte contre le cancer ? », Revue française de gestion, 275(6), 2018, p. 43-58.

Birkinshaw J., Hamel G., Mol M. J., "Management innovation", Academy of management Review, 33(4), 2008, p. 825-845.

Burnel P., «L'impact de la T2A sur la gestion des établissements publics de santé », Journal de gestion et d'économie médicales, vol. 35(2), 2017, p. 67-79.

Claveranne J.P., Christophe P., Repenser les processus à l'hôpital : une méthode au service de la performance, Edition Médica Paris, 2004.

Dumez H., «Qu'est-ce que la recherche qualitative? Problèmes épistémologiques, méthodologiques et de théorisation », Annales des Mines-Gérer et comprendre, No. 2, 2013, p.29-42.

Gombault A., Chapitre 2. La méthode des cas, Dans : P. Roussel \& F. Wacheux (Dir), Management des ressources humaines : Méthodes de recherche en sciences humaines et sociales, Louvain-la-Neuve, Belgique : De Boeck Supérieur, 2005, p. 31-64.

Grenier C., «Proposition d'un modèle d'espaces favorables aux habiletés stratégiques », Journal de gestion et d'économie médicales, 32(1), 2014, p. 3-10.

GRENIER C., IBRAHIM R., «La gouvernance auscultée : entre une gouvernance technique qui prescrit et une gouvernance comme espace de renouvellement des relations et des habiletés à innover », Journal de gestion et d'économie médicales, 33(6), 2015, p. 327-334.

JAOUEN A., TESSIER N., Effet de grossissement et proximité dans les pratiques de GRH des très petites entreprises, in JAOUEN A., TORRES O., Les très petites entreprises : un management de proximité, Paris, Lavoisier, 2008, p. 143-165.

JARZABKOWSKi P., LÊ J., VAN DE VEN A., "Responding to competing strategic demands: How organizing, belonging, and performing paradoxes coevolve", Strategic Organization, 11(3), 2013, p. 245-280.

Josserand P., Perret V., Pratiques organisationnelles du paradoxe. Le paradoxe : Penser et gérer autrement les organisations, Ellipses, 2003, p. 165-187.

LAFAYe C., Berger-Douce S., «Veille stratégique en petite entreprise : proposition de la notion d'intelligence collective entrepreneuriale », Revue de l'Entrepreneuriat, vol. 11(2), 2012, p. 11-30.

Le Roy F., Robert M., Giuliani P., «L'innovation managériale », Revue française de gestion, 6(235), 2013, p. 77-90.

LESCA H., CHOKRON M., «Intelligence collective anticipative pour dirigeants d'entreprise. Retours d'interventions », Revue SIM, vol. 7, n 4, 2002, p. 65-90. 
LESCA H., LeSCA N., « Méthodes heuristiques d'entraînement à la détection des signaux faibles », Revue internationale de Psychosociologie, vol. 15, $\mathrm{n}^{\circ} 37,2009$, p. 135-160.

LEwIS M., "Exploring paradox: Toward a more comprehensive guide", Academy of Management Review, 25(4), 2000, p. 760-776.

NoBre T., «L'innovation managériale à l'hôpital », Revue française de gestion, 6(235), 2013, p. 113-127.

Perret V., Josserand E., Le paradoxe : Penser et gérer autrement les organisations. Ellipses, 2003.

PoOle M.S., VAN DE Ven A.H., "Using Paradox to Build Management and Organizational Theories", Academy of Management Review, 14(4), 1989 , p. $562-578$

Ruellan R., La gouvernance de la Sécurité sociale à partir du plan Juppé de 1995, Vie sociale, 10(2), 2015.

SCHAD J., LEWIS M., RAISCh S., SMith W., "Paradox Research in Management Science: Looking Back to Move Forward", The Academy of Management Annals, vol. 10, 2016, p. 5-64.

SMITH W., LEwIS M., "Toward a theory of paradox: A dynamic equilibrium model of organizing", Academy of Management Review, 36(2), 2011, p. 381-403.

VANDANGEON-Derumez I., AUtissier D., Construire du sens pour réussir les projets de changement, in Les Défis du sensemaking en entreprise, Karl E. Weick et les sciences de gestion, 2006, p. 169-196.

WeICK K.E., Sensemaking in organization, Sage, 1995.

ZEYNEP Or., "Implementation of DRG Payment in France: Issues and recent developments", Health Policy, vol. 117, Issue 2, 2014, p. 146-150. 